\title{
Electrical Percolation, Morphological and Dispersion Properties of MWCNT/PMMA Nanocomposites
}

\author{
Paulo Henrique da Silva Leite Coelho*, Marcel Silva Marchesin, \\ Ana Rita Morales, Julio Roberto Bartoli \\ School of Chemical Engineering, University of Campinas - UNICAMP, \\ Av. Albert Einstein, 500, CEP 13083-852, Campinas, SP, Brazil
}

Received: June 27, 2013; Revised: March 27, 2014

\begin{abstract}
Nanocomposites of poly (methyl methacrylate) (PMMA) and carbon nanotubes have a high potential for applications where conductivity and low specific weight are required. This piece of work concerns investigations of the level of dispersion and morphology on the electrical properties of in situ polymerized nanocomposites in different concentrations of multi-walled carbon nanotubes (MWCNT) in a PMMA matrix. The electrical conductivity was measured by the four point probe. The morphology and dispersion was analyzed by Transmission Electron Microscopy (TEM) and Small Angle X-ray Scattering (SAXS). The correlation between electrical conductivity and the MWCNT amount, presented a typical percolation behavior, whose electrical percolation threshold determined by power law relationship was $0.2 \mathrm{vol}$. (\%) The exponent $t$ from the percolation power law indicated the formation of a 3D network of randomly arranged MWCNT. SAXS detected that the structures are intermediate to disks or spheres indicating fractal geometry for the MWCNT aggregates instead of isolated rods. HR-TEM images allowed us to observe the MWCNT individually dispersed into the matrix, revealing their distribution without preferential space orientation and absence of significant damage to the walls. The combined results of SAXS and HR-TEM suggest that MWCNT into the polymeric matrix might present interconnected aggregates and some dispersed single structures.
\end{abstract}

Keywords: percolation, SAXS, carbon nanotubes, PMMA

\section{Introduction}

Electrically conductive particles, such as metallic powder, graphite, carbon black (CB) and carbon nanotubes (CNT) when added above a critical concentration into an insulating resin have been a useful method to produce conductive composites ${ }^{1}$.

Developing these conductive polymeric composites enables a number of applications previously limited to metals such as piezoelectric materials ${ }^{2}$, conducting adhesives $^{3}$, tissues and antistatic materials ${ }^{4}$, electromagnetic shielding ${ }^{5}$ and chemical sensors ${ }^{6}$.

Some of these applications demand the preparation of electrical conductive Nano composites from insulating polymeric matrices modified by CNT. Unlike conventional spherical particles, carbon fibers or layered silicates, CNT are very lengthy Nano fillers with high values of aspect ratio $(>1000)$ and surface area $\left(>1000 \mathrm{~m}^{2} / \mathrm{g}\right)$. The application of such materials, however, has been limited by the strong Van Der Walls forces among the nanotubes surfaces, favoring the formation of agglomerates and poor dispersion states.

Since the discovery of carbon nanotubes by Iijima ${ }^{7}$ the scientific activities in this field have followed an exponential increase due to the remarkable physical properties of these carbon nanomaterials ${ }^{8,9}$.

CNT can be visualized as a graphen layer, essentially a single sheet of graphene, which has been rolled up to form a

*e-mail: piyke.coelho@gmail.com tube. For this reason CNT inherit has the chemical stability similar to the graphite.

In particular, some mechanical properties of carbon nanotube have been reported to be outstanding. For example, Young Modulus in the range of 1TPa compares to that of a diamond (1.2 TPa), while the tensile strength (40-50 GPa) is in the range of the steel $l^{8,10}$.

Therefore, CNT are expected to be the basis of a new generation of advanced materials, including a new class of polymer nanocomposites ${ }^{11}$.

The electronic properties of single-walled carbon nanotubes (SWCNT) are quite distinct from MWCNT. The chirality associated to the carbon atoms arranged in a single wall allows metallic or semi metallic behavior, which is interesting for optoelectronic research. Aside, a nanotube with several walls, which can be produced in large scale, behaves as a zero-gap metal, which makes the carbon nanotube of interest for applying as a conductive Nano filler in electronic nanomaterials ${ }^{1}$.

A nanocomposite can be defined as a nanomaterial in which the total interfacial phase becomes the critical parameter rather than the volume fraction of the filler ${ }^{11}$ whose properties are governed by the interfacial interactions. The high aspect ratio and nanoscopic scale of carbon nanotubes provide CNT-based nanomaterials whose properties are unobserved in isolated components. 
The dispersion must be interpreted concerning two distinct aspects: first, the agglomerates must be dissociated in order to individualize the CNT; posteriorly, must be distributed randomly in the polymeric matrix ${ }^{12}$.

The main aspect related to introduce conductive properties to an insulating polymer by adding conductive particles, is the relation between the conductivity and the amount of the particles. Thus, above a critical concentration, the conductivity value of the composite rises significantly. This behavior is associated to the formation of an interconnected network of conductive particles as described by the percolation theory ${ }^{13}$. The particle's size and geometry are secondary factors affecting the percolation behavior ${ }^{14}$. The knowledge of the critical percolation concentration of the particles into the Nano composite, and how to minimize it, is relevant to reduce costs as well as to control other properties of the composite.

Several physical models were developed in order to explain this phenomenon and predict the percolation threshold. In an important review done by Lux $^{13}$, these models are discussed considering the microstructural aspects of the mixtures.

According to the classic theory of percolation, the percolation threshold $(p)$ can be obtained by the power law relationship, relating the volumetric electrical conductivity $(\sigma)$ to the volumetric concentration of conductive filler into the matrix $(p)$. The Equation 1 describes the phenomenon:

$\sigma \alpha\left(p-p_{c}\right)^{t}$

Wherein $t$ is the critical exponent and is related to network dimensional aspects.

The real percolation threshold is also dependent on several other properties, such as the chemical nature of the components, connectivity between the phases, preparation method, and filler dispersion among others ${ }^{15}$. This approach reinstates the importance of a morphological analysis of the composite, in order to correlate the preparation variables to the characteristics of the percolation network obtained.

SAXS is particularly interesting for the analysis of the spatial arrangement of CNT in polymers and liquids, on account of the hierarchical nature of the CNT dispersions. This procedure has been applied to study nanocomposites of MWCNT in a thermoplastic polymer ${ }^{16,17}$. The scattering intensity has been shown to follow a power law as:

$\mathrm{I}(\mathrm{q}) \alpha \mathrm{q}^{-\alpha}$

where $q=4 \pi \sin (\theta) / \lambda$ is the scattering vector being $2 \theta$ the scattering angle and $\lambda$ the wavelength of the radiation used.

This type of power law is characteristic of the scattering by fractals ${ }^{16,19}$. Values of $\alpha=1,2,3$ are expected when the scattering objects are rods, discs and spheres, respectively ${ }^{16}$. Intermediate cases where $2<\alpha<3$ are characteristic of objects possessing a fractal geometry ${ }^{18,20-22}$.

In the present work, MWCNT at several concentrations were dispersed into methyl methacrylate monomer (MMA), followed by in situ polymerization, in order to correlate the filler morphology and dispersion to the volumetric electrical conductivity of the obtained MWCNT/ PMMA nanocomposites. The percolation threshold of the nanocomposite volumetric conductivity was determined by applying the power law relationship. Morphological and dispersion characteristics of the conductive filler in the polymeric matrix were discussed for nanocomposites formulations containing loads of MWCNT close to the percolation threshold as well as close to the conductivity limit.

\section{Experimental}

\subsection{Material and methods}

The MWCNT employed was the TNM2 from Chengdu Organic Chemicals Co. Ltd fabricated by chemical vapor deposition with $95 \%$ of purity. The monomer was the MMA M/4950/08 from Fischer Scientific stabilized with hydroquinone. The solvent employed was acetone PA-ACS from QUEMIS. The polymerization was conducted in the presence of the initiator 2,2'-Azobys(2-methylpropionitrile) (AIBN) Vaso 64, DuPont.

The monomer was previously purified using a 311332 column of Sigma Aldrich to remove hydroquinone. In sequence, water traces were removed with magnesium sulfate. Pre mixtures were prepared by adding different concentrations of MWCNT into glass tubes containing $6 \mathrm{ml}$ of acetone, $3 \mathrm{ml}$ of MMA, and $16.7 \mathrm{mmol} . \mathrm{L}^{-1}$ of AIBN. The solutions were purged with $\mathrm{N}_{2}$, and then submitted to ultrasonic stirring using a UP400S probe, by Hielscher, power of $100 \mathrm{~W}$ and constant pulse frequency of $24 \mathrm{KHz}$ for 20 minutes. The polymerization processes of the sonicated solutions were completed after 24 hours at $80{ }^{\circ} \mathrm{C}$. The obtained products were black and homogeneous liquids used as precursors to make polymeric films by casting on a PTFE circular planar substrate, at room temperature. Samples with $0.5 \pm 0.1 \mathrm{~mm}$ of thickness and $55 \mathrm{~mm}$ in diameter were subsequently placed in an oven at $80{ }^{\circ} \mathrm{C}$ for $24 \mathrm{~h}$ for further solvent removal. To ensure complete removal of the solvent TGA and DSC analyses were performed The volume fractions of the polymerized components were determined after considering the density of MWCNT, measured by gaseous pycnometer (Equipment AccuPyc 1330 - Sample Weight $=1.4374 \mathrm{~g}$ and $\mathrm{T}=28.6^{\circ} \mathrm{C}$ ), and the density of PMMA measured by liquid pycnometry ${ }^{23}$. The nanocomposites formulations were prepared as shown in Table 1.

\subsection{Volumetric electrical conductivity measurements}

The volumetric electrical conductivity of MWCNT/ PMMA films was determined by a four point probe test, Jandel Universal Probe RM3, supplied with a $100 \mathrm{ohm}$ test resistor and with aligned probes and spacing of $0.1 \mathrm{~cm}$. Measurements were made in the range of $10 \mathrm{nA}-10 \mu \mathrm{A}$.

\subsection{Morphological analysis}

High resolution transmission electron microscopy (HR-TEM) analysis was performed using a JEOL 3010 (tension of $300 \mathrm{kV}$ ) on ultra-thin film sections of the sample containing 0.62 vol. (\%) of MWCNT. Films with an approximate thickness of $40 \mathrm{~nm}$ were obtained using an Ultra microtome LEICA model ULTRACUT - UCT 020 , at room temperature and cutting speed of $1 \mathrm{~mm} / \mathrm{s}$. 
Table 1. Weight and volume loads of MWCNT in the nanocomposites of PMMA.

\begin{tabular}{ccc}
\hline Samples & MWCNT wt. (\%) & MWCNT vol. (\%) \\
\hline 1 & 0.1 & 0.06 \\
2 & 0.2 & 0.11 \\
3 & 0.3 & 0.17 \\
4 & 0.4 & 0.22 \\
5 & 0.5 & 0.28 \\
6 & 0.6 & 0.34 \\
7 & 0.8 & 0.45 \\
8 & 0.9 & 0.51 \\
9 & 1.0 & 0.56 \\
10 & 1.1 & 0.62 \\
11 & 1.2 & 0.67 \\
12 & 1.3 & 0.73 \\
13 & 2.0 & 1.13 \\
14 & 4.0 & 2.28 \\
\hline
\end{tabular}

The microscopy analyses were done at the Brazilian Nanotechnology Laboratory - LNNano.

\subsection{Small-angle $X$-ray scattering}

SAXS analysis were performed on the D01ASAXS1 beam line of the Brazilian Synchrotron Light Laboratory $(\mathrm{LNLS})^{24}$. SAXS experimental sections were done on two runs, according to the distance between detector and sample cell, incorporated in a vacuum chamber of $3.5 \times 10^{-2}$ torr, at room temperature. The scattered beam $(\lambda=0.1488 \mathrm{~nm})$ was detected in a Pilatus $300 \mathrm{k}$ area detector placed, for SAXS, at $1300 \mathrm{~mm}$ away from the sample. A polyimide film (Kapton) was used for calibration (time $100 \mathrm{~s}$ ). Each sample frame was collected during $5 \mathrm{~s}$.

\section{Results and Discussion}

\subsection{Volumetric electrical conductivity}

Figure 1 shows the electrical conductivity of MWCNT/ PMMA nanocomposites samples as a function of volumetric concentration of MWCNT. It was not observed any change on conductivity for systems with 0.06 to 0.17 vol. (\%) of MWCNT. The conductivity values in this range were around $10^{-11} \mathrm{~S} / \mathrm{cm}$, typical of dielectric materials. On the other hand, MWCNT/PMMA nanocomposites containing loads of Nano fillers of $0.22 \mathrm{vol}$. (\%) exhibits an expressive increase on its electrical conductivity, reaching values between $10^{-5}$ and $10^{-6} \mathrm{~S} / \mathrm{cm}$. The conductivity values continue to increase for higher loads of MWCNT to $10^{-1} \mathrm{~S} / \mathrm{cm}$ with $1.13 \mathrm{vol}$. (\%) of MWCNT. Such an increase corresponds to a rise of 10 orders of magnitude in comparison to the low loaded samples. Small changes in volume fraction of conductive particle cause high changes on electrical conductivity, characterizing its percolation curve. The range between $0.1-10 \mathrm{~S} / \mathrm{cm}$ was previously related for semiconductor composites of CNT dispersions in PMMA matrix ${ }^{25-27}$.

In order to determine the critical percolation threshold on electrical conductivity, it was applied the power law

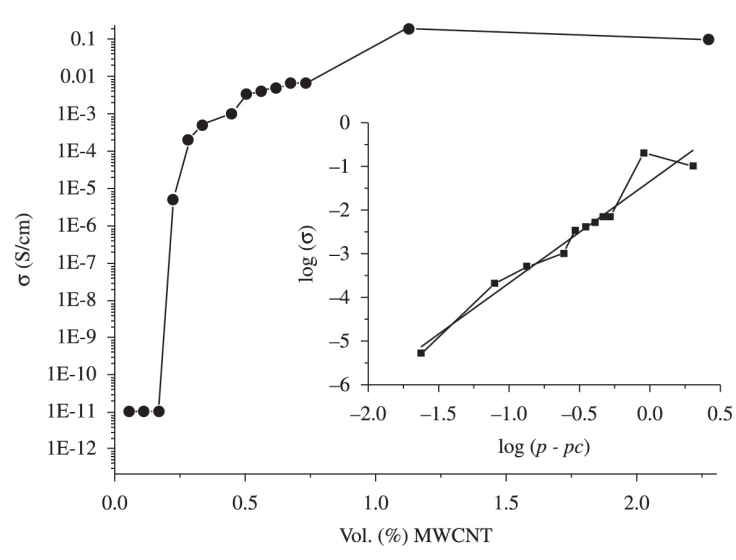

Figure 1. Volumetric electrical conductivity as a function of MWCNT concentration for MWCNT/PMMA nanocomposites.

relationship, according to the classical model of percolation theory, described in Equation 1. Experimental results were analyzed based on the double logarithmic graphic $\log \sigma v s$. $\log \sigma\left(p-p_{c}\right)$ (Figure 1) varying the value of $p_{c}$ until the best linear fit was obtained.

The experimental $p_{c}$ value was 0.20 vol. (\%) of MWCNT, which was in accordance to other studies discussed in the literature for MWCNT and PMMA ${ }^{25}$. The critical exponent $t$ is dependent on the system dimension and is set between 1.65 and 2 for three-dimensional network ${ }^{28-30}$. However, for CNT-polymer composites, the range of critical exponent values fitted from experimental measurements obtained by different situations clearly indicates that the $t$ is not universal. Bauhofer and Kovacs ${ }^{1}$ have indicated $\mathrm{t}$ values obtained from experiments for three-dimensional percolating systems between 1.3 and 4 . Other studies have also shown that the percolation threshold is dependent of conductive fillers morphology (size, shape and the aspect ratio $)^{30-33}$. In this piece of work, the value of $t$ obtained was $2.34 \pm 0.19$ for a regression coefficient of 0.94 , which is evidence of a three-dimensional conductive network of MWCNT into the polymer matrix. Kim et al. ${ }^{26}$ related critical exponent $t$ of 2.4 and $p_{c}=0.3 \mathrm{wt} \%$ for MWCNT/ PMMA composites.

\subsection{Morphological analysis}

The images obtained by HR-TEM (Figure 2a and b) are generated due to the contrast that arises from the interference in the transmittance of the electron beam by regions with different density of carbon atoms of the very thin sample nanocomposites. The electron beam is scattered more intensely in thicker regions of the sample, which corresponds to darker areas on micrograph, and mostly by atomic columns, which appears as dark lines associated to scattering generated by the interaction of the beam at the tangent of the tubes walls. Examining the parallel section of the MWCNT in Figure 2a it is possible to observe that the integrity of the walls of the carbon nanotubes are well preserved, even after being cut by the blade. This could indicate that the sonication processes and the in situ polymerization had little effect on the generation of 
structural defects preserving the conductive properties of the Nano fillers. In Figure $2 b$ one can identify circular patterns with a bright center corresponding to Nano fillers arranged perpendicularly to the cutting direction. The extended and overlapping patterns are related to a nanotube parallel to the cutting plane and give evidence of their interconnectivity. Such behavior suggests that the bulk of material is composed by single nanotubes randomly arranged and showing some interconnectivity among them, in a three dimensional (3D) structure. The maximum tube external diameter detected in Figure $2 \mathrm{c}$ and $\mathrm{d}$ was around $30 \mathrm{~nm}$.

\subsection{Small-angle $X$-ray scattering}

Figure 3 shows the SAXS scattered intensity as a function of the scattering vector $q$ for the MWCNT/PMMA nanocomposites, whose MWCNT volume concentration has been labeled on the right. The results are plotted in a double logarithmic scale in order to fit the power law relationship. It is observed a value of $\alpha=2.96$ for the $0.06 \mathrm{vol}$. (\%) of MWNCT, that is preceding electrical percolation where, probably, the scattering entities behavior like spheres, and $\alpha$ decreases to 2.46 for the higher concentration of filler 0.62 vol. (\%) likely something between spheres and discs,

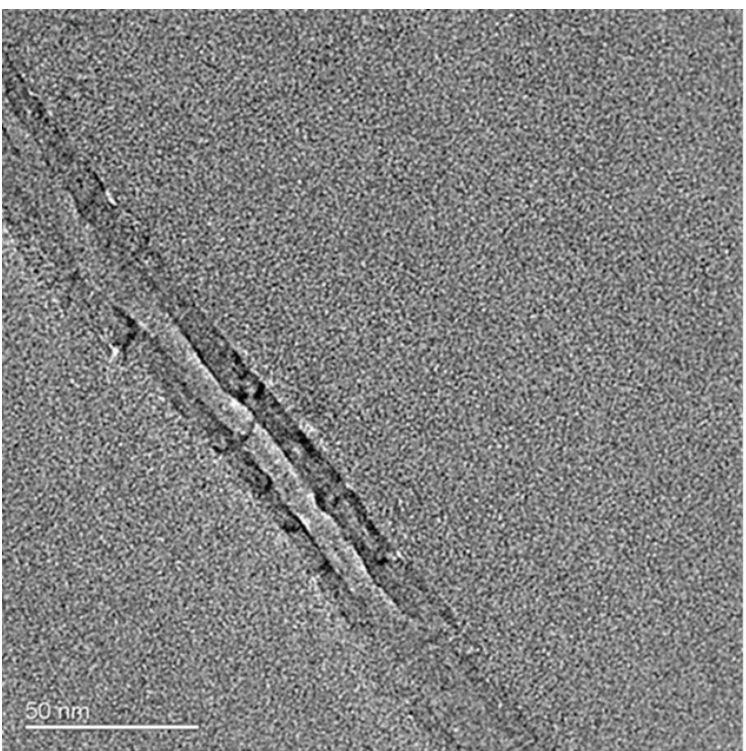

(a)

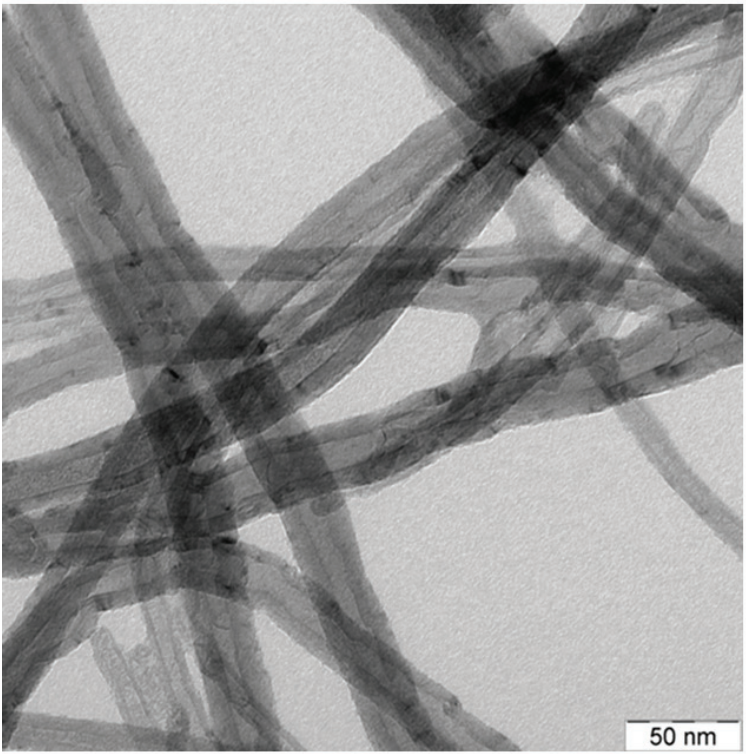

(c)

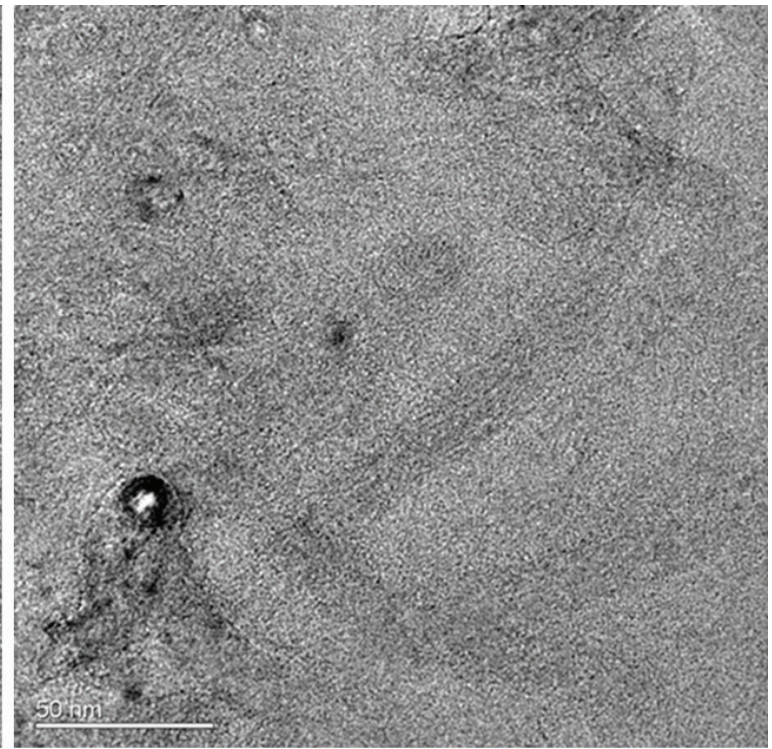

(b)

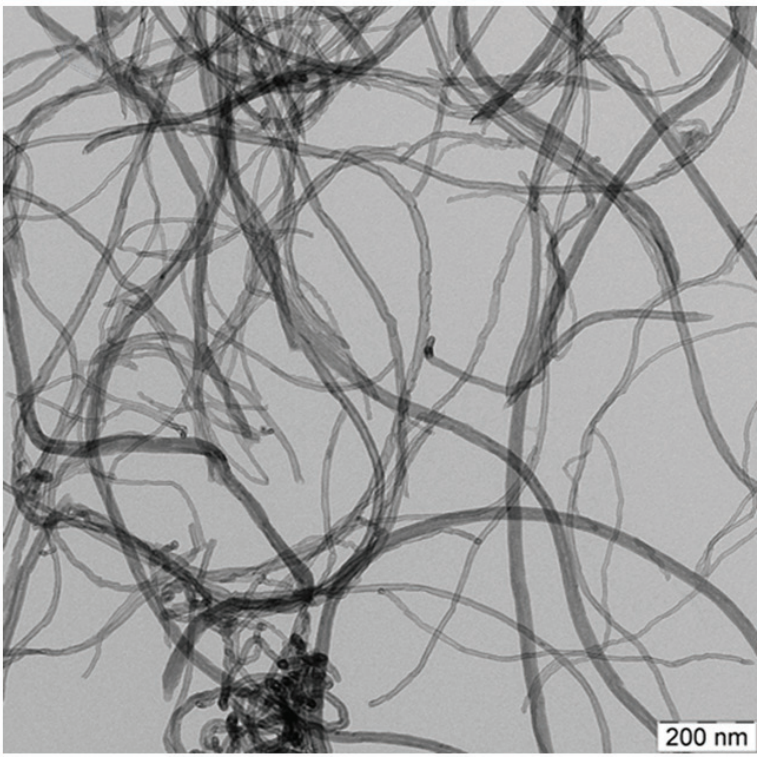

(d)

Figure 2. TEM micrographs showing nanotubes: (a) parallel to the cutting direction for 0.62MWCNT/PMMA; (b) random cutting direction for 0.62MWCNT /PMMA; (c) and (d) for pure MWCNT. 


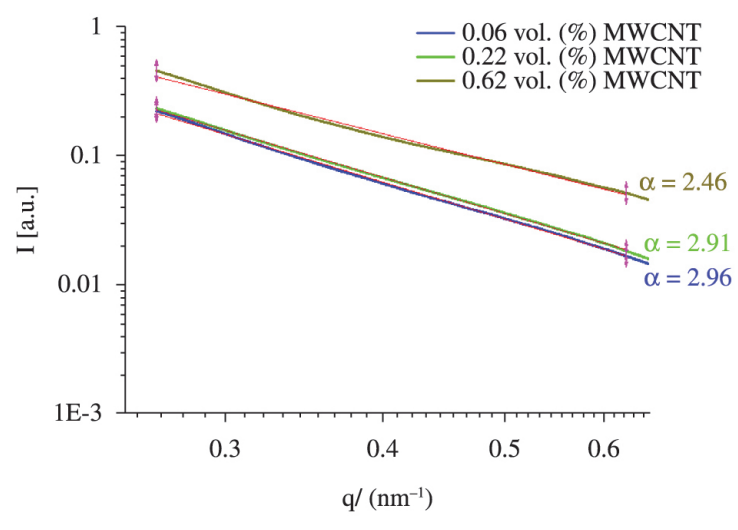

Figure 3. SAXS intensity as a function of the scattered q-vector plotted in double logarithmic scale for nanocomposites of MWCNT/ PMMA.

as well some single nanotubes dispersed, as shown by HRTEM. The slope values of $\alpha$ from 2.5 to $<3.0$ are indicating that MWCNT aggregates form fractal structures, i.e. the entity that causes the scattering are clusters of entangled nanotubes, as observed by other authors ${ }^{16,20}$. Therefore, both SAXS and HR-TEM analyses showed MWCNT aggregates and some interconnected dispersed single structures into the PMMA matrix. In addition, these slope values point out the absence of only individual rod-like scatters which would produce slope of $\alpha=1^{16}$.

Percolation theory indicates the $\mathrm{t}$ exponent of $3 \mathrm{D}$ structure, suggesting the interconnectivity of the MWCNT aggregates turning the system electrically conductive, supported by the SAXS analyses showing a quasi 3D structure. It could be, that prior to the percolation, the aggregates could be similar to isolated spheres and for

\section{References}

1. Bauhofer $\mathrm{W}$ and Kovacs JZ. A review and analysis of electrical percolation in carbon nanotube polymer composites. Composites Science and Technology. 2009; 69(10):1486-1498. http://dx.doi.org/10.1016/j.compscitech.2008.06.018

2. Hori M, Aoki T, Ohira Y and Yano S. New type of mechanical damping composites composed of piezoelectric ceramics, carbon black and epoxy resin. Composites Part A: Applied Science and Manufacturing. 2001; 32(2):287-290. http:// dx.doi.org/10.1016/S1359-835X(00)00141-X

3. Marcq F, Demont P, Monfraix P, Peigney A, Laurent Ch, Falact $\mathrm{T}$ et al. Carbon nanotubes and silver flakes filled epoxy resin for new hybrid conductive adhesives. Microelectronics Reliability. 2011; 51(7):1230-1234. http://dx.doi.org/10.1016/j. microrel.2011.03.020

4. Li C, Liang T, Lu W, Tang C, Hu X, Cao M et al. Improving the antistatic ability of polypropylene fibers by inner antistatic agent filled with carbon nanotubes. Composites Science and Technology. 2004; 64(13-14):2089-2096. http://dx.doi. org/10.1016/j.compscitech.2004.03.010

5. Chung DDL. Review: electromagnetic interference shielding effectiveness of carbon materials. Carbon. 2001; 39(2):279285. http://dx.doi.org/10.1016/S0008-6223(00)00184-6 a higher concentration of filler, a network structure of individuals dispersed MWCNT prevalence than aggregate fillers. Therefore, SAXS analyses agrees with the proposed model suggesting intermediate structures between aggregates spheres (3D) and totally dispersed fillers (1D), describing the fractal structure.

\section{Conclusion}

The preparation of conductive nanocomposites by in situ polymerization of MWCNT/PMMA showed to be an efficient method to increase the volumetric electrical conductivity of the matrix up to 10 orders of magnitude, with values of $10^{-4}$ to $10^{-3} \mathrm{~S} / \mathrm{cm}$, making off the dielectric PMMA into a semiconductor. The power law percolation model was accurate to determine the critical concentration. It was determined a percolation threshold of 0.2 vol. (\%) of MWCNT for the studied nanocomposites. The HR-TEM morphological analysis suggest that the preparation method (sonication and in situ polymerization processes) preserves the integrity of the MWCNT walls, as few structural defects were generated, and so preserving the conductive properties of the nanoparticles. SAXS analyses indicate an intermediate structure between aggregates spheres (3D) and totally dispersed fillers (1D), describing the fractal structure and that interconnectivity is necessary to form a conducting percolation network.

\section{Acknowledgements}

The research was conducted with support from LNNano - LNLS- Brazilian Synchrotron Light Laboratory, CNPEM/ABTLuS/MCTI. The authors also thank Prof. P.C.M.L. Miranda (IQ/Unicamp); J.Bressanin, R.R.Cecci (FEQ/Unicamp); CNPq for financial support for the project and Semiconducting Components Center (CCS-Unicamp).

6. Sanjinésa R, Abadb MD, Vâjua CR, Smajdaa R, Mionića $\mathrm{M}$ and Magreza A. Electrical properties and applications of carbon based nanocomposite materials: an overview. Surface and Coatings Technology. 2011; 206(4):727-733. http://dx.doi. org/10.1016/j.surfcoat.2011.01.025

7. IIjima S. Helical microtubules of graphitic carbon. Nature. 1991; 354:56-58. http://dx.doi.org/10.1038/354056a0

8. Treacy MMJ, Ebbesen TW and Gibson JM. Exceptionally high Young's modulus observed for individual carbon nanotubes. Nature. 1996; 381:678-680. http://dx.doi. org/10.1038/381678a0

9. Saito R, Dresselhaus G and Dresselhaus MS. Physical properties of carbon nanotubes. London: Imperial College Press; 1998.

10. Wei C, Cho K and Srivastava D. Tensile strength of carbon nanotubes under realistic temperature and strain rate. Physical Review B. 2003; 67(11):115407-115412. http://dx.doi. org/10.1103/PhysRevB.67.115407

11. Vaia RA and Wagner HD. Framework for nanocomposites. Materials Today. 2004; 7(11):32-37. http://dx.doi.org/10.1016/ S1369-7021(04)00506-1

12. Ma PC, Siddiqui NA, Marom GJ and Kim K. Dispersion and functionalization of carbon nanotubes for polymer-based 
nanocomposites: a review. Composites Part A: Applied Science and Manufacturing. 2010; 41(10):1345-1367. http://dx.doi. org/10.1016/j.compositesa.2010.07.003

13. Lux F. Models proposed to explain the electrical conductivity of mixtures made of conductive and insulating materials. Journal of Materials Science. 1993; 28(2):285-301. http:// dx.doi.org/10.1007/BF00357799

14. Strümpler R and Glatz-Reichenback J. Conducting polymers composites. Journal of Electroceramics. 1999; 3(4):329-346. http://dx.doi.org/10.1023/A:1009909812823

15. Ponomarenko AT, Shevchenko VG and Enikolopyan NS. Formation processes and properties of conducting polymer composites. Advances in Polymer Science. 1990; 96:125-147. http://dx.doi.org/10.1007/3-540-52791-5_4

16. Hernández JJ, García-Gutiérrez MC, Nogales A, Rueda DR and Ezquerra TA. Small-angle X-ray scattering of single-wall carbon nanotubes dispersed in molten poly(ethylene terephthalate). Composites Science and Technology. 2006; 66(15):2629-2632. http://dx.doi.org/10.1016/j.compscitech.2006.05.008

17. García-Gutiérrez MC, Nogales A, Hernández JJ, Rueda DR and Ezquerra TA. X-ray scattering applied to the analysis of carbon nanotubes, polymers and nanocomposites. Óptica Pura y Aplicada. 2007; 40(2):195-205.

18. Martin JE and Hurd AJ. Scattering from fractals. Journal of Applied Crystallography. 1987; 20(2):61-78. http://dx.doi. org/10.1107/S0021889887087107

19. Rols S, Almairac R, Henrard L, Anglaret, E and Sauvajol JL. Diameter distribution of single wall carbon nanotubes in nanobundles. European. Physical Journal B. 2000; 18(2):201205. http://dx.doi.org/10.1007/s100510070049

20. Bauer BJ, Hobbie EK and Becker ML. Small-angle neutron scattering from labeled single-wall carbon nanotubes. Macromolecules. 2006; 39(7):2637-2642. http://dx.doi. org/10.1021/ma0527303

21. Schaefer DW, Zhao J, Brown JM, Anderson DP and Tomlin DW. Morphology of dispersed carbon single-walled nanotubes. Chemical Physics Letters. 2003; 375(3-4):369-375. http:// dx.doi.org/10.1016/S0009-2614(03)00867-4

22. Zhou W, Islam MF, Wang H, Ho DL, Yodh AG, Winey KI et al. Small angle neutron scattering from single-wall carbon nanotube suspensions: evidence for isolated rigid rods and rod networks. Chemical Physics Letters. 2004; 384:185-189. http://dx.doi.org/10.1016/j.cplett.2003.11.106

23. César J, Paoli MA and Andrade JCA. Determinação da densidade de sólidos e líquidos. Chemkeys. 2004;1-8.
24. Westfahl H Jr and Cardoso MB. Accessing the hidden lamellar nanostructure of semi-crystalline nascent polymers by smallangle X-ray scattering contrast variation. Journal of Applied Crystallography. 2011; 44(5):1123-1126. http://dx.doi. org/10.1107/S0021889811033255

25. Stephan C, Nguywn TP, Lahr B, Blau W, Lefrant S and Chauvet O. Raman spectroscopy and conductivity measurements on polymer-multiwalled carbon nanotubes composites. Journal of Materials Research. 2002; 17(2):396-400. http://dx.doi. org/10.1557/JMR.2002.0055

26. Kim HM, Kim K, Lee SJ, Joo J, Yoon HS and Cho SJ. Charge transport properties of composites of multiwalled carbon nanotube with metal catalyst and polymer: application to electromagnetic interference shielding. Current Applied Physics. 2004; 4(6):577-580. http://dx.doi.org/10.1016/j. cap.2004.01.022

27. Sundaray B, Subramanian V, Natarajan TS and Krishnamurthy $\mathrm{K}$. Electrical conductivity of a single electrospun fiber of poly (methyl methacrylate) and multiwalled carbon nanotube nanocomposite. Applied Physics Letters. 2006; 88(14):143114143116. http://dx.doi.org/10.1063/1.2193462

28. Balberg I. Tunneling and nonuniversal conductivity in composite materials. Physical Review Letters. 1987; 59(12):13051308. PMid:10035198. http://dx.doi.org/10.1103/ PhysRevLett.59.1305

29. Newnham RE, Skinner DP and Cross LE. Connectivity and piezoelectric-pyroelectric composites. Materials Research Bulletin. 1978; 13(5):525-536. http://dx.doi.org/10.1016/00255408(78)90161-7

30. Kirkpatrick S. Percolation and conduction. Reviews and Modern Physics. 1973; 45(4):574-588. http://dx.doi. org/10.1103/RevModPhys.45.574

31. Balberg I and Binenbaum N. Computer study of the percolation threshold in a two-dimensional anisotropic system of conducting sticks. Physical Review B. 1983; 28:3799-3812. http://dx.doi.org/10.1103/PhysRevB.28.3799

32. Heo SI, Yun C, Oh KS and Han KS. Influence of particle size and shape on electrical and mechanical properties of graphite reinforced conductive polymer composites for the bipolar plate of PEM fuel cells. Advanced Composite Materials. 2006; 15;115126. http://dx.doi.org/10.1163/156855106776829356

33. Celzard A, McRae E, Deleuze C, Dufort M, Furdin G and Marêché JF. Critical concentration in percolating systems containing a high-aspect-ratio filler. Physical Review B. 1996; 53:6209-6214. http://dx.doi.org/10.1103/ PhysRevB.53.6209 